\title{
Impact of conscious intent on chunking during motor learning
}

\author{
Sunbin Song and Leonardo Cohen
}

Human Cortical Physiology and Stroke Neurorehabilitation Section, National Institute of Neurological Disorders and Stroke, National Institutes of Health, Bethesda, Maryland 20892, USA

\begin{abstract}
Humans and other mammals learn sequences of movements by splitting them into smaller "chunks." Such chunks are defined by the faster speed of performance of groups of movements. The purpose of this report is to determine how conscious intent to learn impacts chunking, an issue that remains unknown. Here, we studied 80 subjects who either with or without conscious intent learned a motor sequence. Performance was tested before and up to 1-wk post-training. Chunk formation, carryover of chunks, and concatenation of chunks into longer chunks, all measures of motor chunking success, were determined at each time-point. We found that formation, carryover, and concatenation of chunks were comparable across groups and did not improve over the training session and subsequent testing times. Thus, motor learning progressed in the absence of improvements in chunking irrespective of conscious intent. These data suggest that mechanisms other than chunking contribute to successful motor learning with and without conscious intent.
\end{abstract}

[Supplemental material is available for this article.]

Human skills such as speech or playing a musical instrument rely on our ability to learn long and complex movement sequences (Lashley 1951) both with and without conscious intent to learn (Keele et al. 2003; Song 2009). One early and influential theoretical account for how we learn is through "chunking" (Miller 1956), which refers to a process in which a long and complex sequence is broken down into more manageable fragments that can be learned and then concatenated together to acquire the long sequence. Chunking provides a conceptual framework upon which researchers have come to understand how humans and other mammals learn motor skills (Graybiel 1998).

In prior studies where learning occurred with conscious intent to learn, motor chunks, defined by faster speed of performance of groups of movements on average three to four items in length (Rosenbaum et al. 1983), were unique for each person (Sakai et al. 2003; Kennerley et al. 2004), and at the end of training had lengths that correlated with working memory capacity (Bo et al. 2009). In situations in which learning was without conscious intent, rearranging the long sequence by disrupting items within chunks was more harmful than disruptions between chunks (Koch and Hoffmann 2000). However, a comparison between learning with and learning without conscious intent has not been experimentally tested.

Thus, although chunking is thought to be necessary for overcoming the limited number of items humans can consciously hold at any given time within working memory (Miller 1956), the impact of conscious intent on chunking remains unknown. This information may be important to optimize learning strategies in different contexts and in pathological settings (Boyd and Winstein 2006; Song and Cohen 2014b).

Here, we used a novel data-driven strategy to identify motor chunks (k-means clustering [Fig. 1C; Supplemental Material]) that does not require a priori assumptions on chunk characteristics (i.e., chunk lengths) and determined the influence of conscious intent on chunking. After motor chunks were identified, we quantified chunk formation, carryover of chunks, and concatenation

Corresponding authors: songss@mail.nih.gov, cohenl@ninds.nih.gov Article is online at http://www.learnmem.org/cgi/doi/10.1101/lm.035824.114. of chunks, all measures of success of motor chunking (Fig. 2). As motor chunking improved for the 12-item pattern, all measures would approach one. This information was compared to those obtained by calculating these same measures for random blocks, which represent chance levels.

For chunk formation, as motor chunks formed and became stable, the correlation between motor chunks in one repetition of the 12-item pattern and motor chunks in the next repetition should become perfect and approach one. A $2 \times 2 \times 2 \times 10$ Intent $\times$ TOD $\times$ Pattern $\times$ Time-point $\mathrm{ANOVA}_{\mathrm{MD}}$ on correlation values $\left(R^{2}\right)$ of motor chunks between repetitions within a block revealed a significant main effect of Pattern, $F_{(1,75)}=204.4, P<$ 0.0001 , and no significant interactions (Fig. 2A). Thus, irrespective of conscious intent, there was greater than chance formation of pattern-specific chunks that did not increase over training and testing times.

For carryover of chunks, as formed chunks were encoded and carried over across training and testing times, the correlation between the motor chunks in a pattern block at one time-point and motor chunks in a pattern block from the prior time-point should become perfect and approach one. A $2 \times 2 \times 2 \times 10$ Intent $\times$ TOD $\times$ Pattern $\times$ Time-point $\mathrm{ANOVA}_{\mathrm{MD}}$ on correlation values $\left(R^{2}\right)$ across time-points showed a main effect of Pattern, $F_{(1,75)}=307.8, P<0.0001$, and no significant interactions (Fig. 2B). Thus, again irrespective of conscious intent, there was a greater than chance carryover of pattern-specific chunks that did not increase across training and testing times.

For concatenation of chunks, as formed chunks concatenated into longer chunks, the ratio of maximum motor chunk length to total number items within motor chunks should increase and approach one (fully concatenated). A $2 \times 2 \times 2 \times 10$ Intent $\times$ TOD $\times$ Pattern $\times$ Time-point ANOVA $_{M D}$ on the ratio of maximum chunk length to total number of items within chunks did not show any significant main effects nor interactions (Fig. 2C).

This article is distributed exclusively by Cold Spring Harbor Laboratory Press for the first 12 months after the full-issue publication date (see http://learnmem. cshlp.org/site/misc/terms.xhtml). After 12 months, it is available under a Creative Commons License (Attribution-NonCommercial 4.0 International), as described at http://creativecommons.org/licenses/by-nc/4.0/. 
Irrespective of conscious intent, concatenation of pattern-specific chunks remained at chance levels.

Hence, although all subjects learned over the training session and subsequent testing times with superior learning with conscious intent (Fig. 1B), on the other hand, chunking was comparable and did not improve irrespective of whether learning had taken place with or without conscious intent as measured by patternspecific chunk formation, carryover, and concatenation (Fig. 2).

Learning in this task involves performance improvements over the course of training often comparable with and without conscious intent and of additional sleep-dependent gains when conscious intent is present (Robertson et al. 2004; Spencer et al. 2006; Song and Cohen 2014a). It has been proposed that chunking is necessary to overcome working memory limitations during learning (Miller 1956; Wymbs et al. 2012). Additionally, conscious learning of a motor sequence is working memorydependent (Song et al. 2009), and chunk lengths correlate with working memory measures (Bo et al. 2009).

Here, we explored the extent to which conscious intent influenced chunking, and found that it did not affect any aspect of chunking success over training and subsequent testing times in spite of successful learning. Thus our data are consistent with
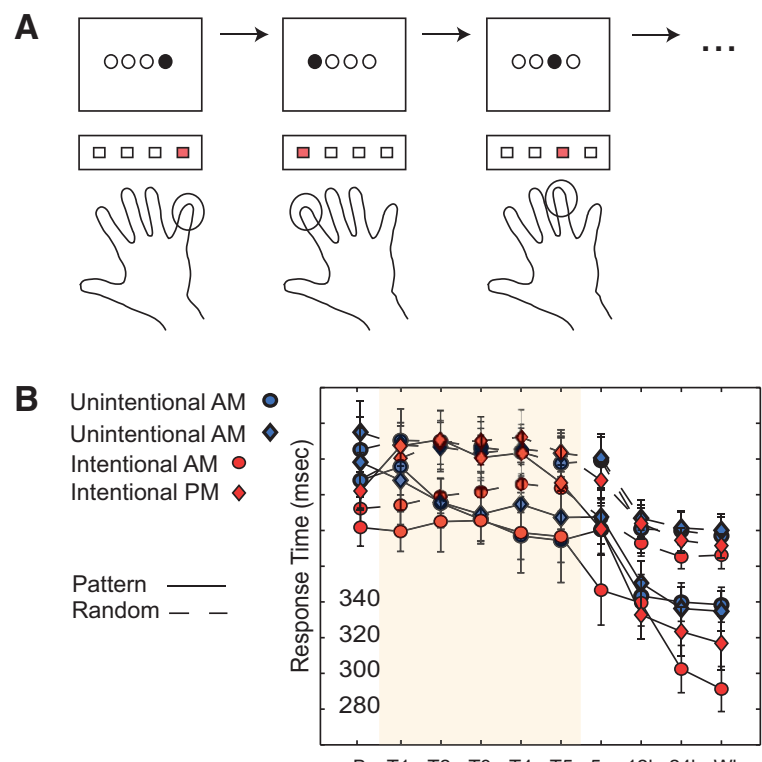

$\begin{array}{lllllllll}\text { B } & \text { T1 } & \text { T2 } & \text { T3 } & \text { T4 } & \text { T5 } & 5 \mathrm{~m} 12 \mathrm{~h} 24 \mathrm{~h} \mathrm{Wk}\end{array}$
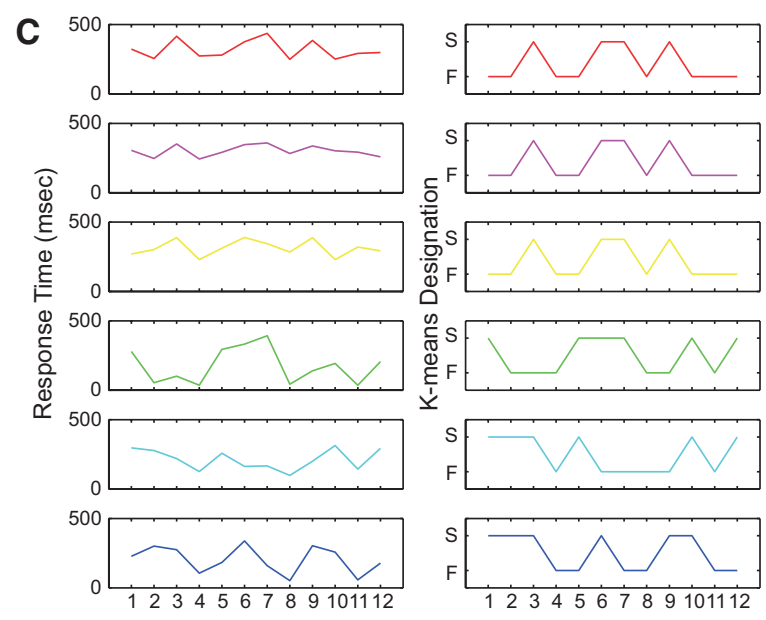

Key-press in 12-item patterm the view that mechanisms other than chunking are contributing to learning with conscious intent. Consistently, Bo et al. (2009) was able to correlate working memory with chunk lengths but not with learning measures. A possible contributing mechanism may be sleep-dependent processes present in learning with conscious intent (Robertson et al. 2004; Spencer et al. 2006; Song and Cohen 2014a). Specifically, it was demonstrated that a sequence encountered during the day is replayed at a condensed rate during sleep to enhance offline learning (Skaggs and McNaughton 1996). In learning without conscious intent, our results are consistent with those of Jimenez (2008) who also found learning could proceed in the absence of improvements in chunking. Additionally, learning frequencies of movement to movement transitions occur both with and without conscious intent (Song et al. 2007a,b; Song and Cohen 2014a) and, though models posit that higher frequency transitions grab more attention and facilitate the choice of chunks that contain high frequency transitions (Perruchet and Vinter 2002; Perruchet et al. 2002), transition learning can also be chunking-independent such as in recursive reinforcement learning (Murray et al. 2011). Thus, previous and present results suggest that learning both with and without conscious intent does not require chunking improvements.

Though further study is necessary, our findings indicate that chunking alone cannot explain all learning both with and without conscious intent, and other potential mechanisms (Skaggs and McNaughton 1996; Murray et al. 2011) can play contributing roles. This is an issue for future investigation with implications for the design of training and rehabilitation strategies and enhanced learning.

Figure 1. Motor learning. $(A)$ In this task (Goedert and Willingham 2002), a circle was filled in (the target) and subjects made a key-press response with the corresponding finger of the right hand, after which another target appeared. Targets followed a 12-item pattern in Pattern blocks, and were randomly ordered in Random blocks (for more detail, see Song and Cohen 2014a). (B) Motor learning: Increasing differentials in response times (RTs) between Pattern (solid lines) and Random (dashed lines) blocks demonstrates motor learning in both groups split by TOD (time-of-day). Half of the subjects in each instructional group were tested in the a.m. and half in the p.m. This was done to control for circadian influences and to account for sleep-dependent processes present in learning with conscious intent that occur between 12- and 24-h delays in the a.m. group and between 5-min and 12-h delays in the p.m. group (Robertson et al. 2004; Spencer et al. 2006; Song and Cohen 2014a). The intentional groups (red) showed more learning compared to the unintentional groups (blue) irrespective of TOD. This was confirmed by a $2 \times 2 \times 2 \times 10$ Intent $\times$ TOD $\times$ Pattern $\times$ Time-point ANOVA $_{M D}$ (significant main effects of Pattern, $F_{(1,75)}=92.6, P<$ 0.0001 ; Time-point, $F_{(4.1,304.6)}=77.7, P<0.0001$, and significant interactions of Intent by Time-point, $F_{(4.1,304.6)}=3.7, P<0.006$; Pattern by Time-point, $F_{(4.3,318.8)}=10.0, P<0.0001$; and Intent by Pattern by Time-point, $\left.F_{(4.3,318.8)}=3.5, P<0.008\right)$. Pattern and Random blocks were interleaved during training, which improves long-term learning (Song et al. 2012). Note that during training (shaded orange), concurrent explicit learning in the Intentional groups can slow down performance as has been previously described (Song et al. 2009). RTs are again plotted, but for each group separately, in Supplemental Figure S1. (C) Motor chunks: Each Pattern block contained eight repetitions of a 12-item pattern (a total of 96 key-presses). (Left) Each row depicted here represents the response times (RTs) for a single repetition of the 12-item pattern with the 12 key-presses arranged left to right on the $x$-axis. The RTs for the 12 key-presses self-sorted as either fast (F) or slow (S) with k-means clustering as depicted to the right. After k-means clustering, motor chunks were easily visible as groups of fast items. In the example used here, the top three rows are taken from three repetitions in the baseline Pattern block and the bottom three rows are taken from three repetitions in the 1-wk Pattern block. Note that even though RTs decrease significantly from baseline to $1 \mathrm{wk}$ as seen on the left, only the relative RT of each item to another item within a single repetition of the 12 -item pattern is important for motor chunk identification as seen on the right. 
A

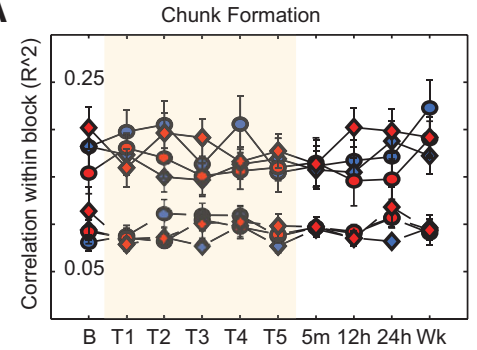

B

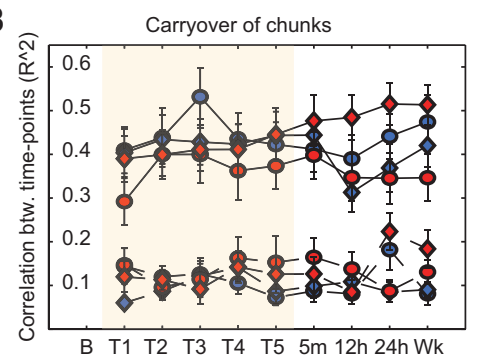

C

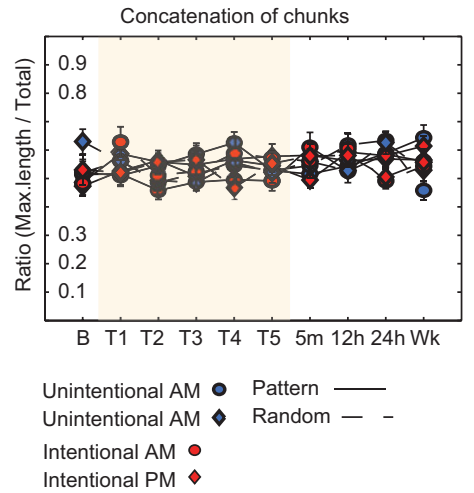

Figure 2. Motor chunking. (A) Chunk formation. As motor chunks formed and became stable, the correlation between motor chunks in one repetition of the 12-item pattern and motor chunks in the next repetition should become perfect and approach one, as whatever is fast (F) in one repetition should be fast in the next repetition and whatever is slow $(S)$ in one repetition should be slow in the next repetition. Hence, we correlated motor chunks in each repetition with those of the next repetition within a block (Pearson's $R^{2}$ ) and averaged these seven $R^{2}$ values (first to second repetition, second to third repetition, etc., yields seven values) to obtain a measure of chunk formation. Irrespective of conscious intent, there was greater than chance formation of pattern-specific chunks that did not increase over training and testing times. (B) Carryover of chunks. As formed chunks were encoded and carried over across training and testing times, the correlation between the motor chunks in a pattern block at one time-point and motor chunks in a pattern block from the prior time-point should become perfect and approach one, as whatever is generally fast ( $\mathrm{OF}$ to $8 \mathrm{~F}$ ) at one time-point should have been generally fast $(\mathrm{OF}$ to $8 \mathrm{~F})$ in the prior time-point. Hence, we correlated motor chunks in each time-point with that of the prior time-point (i.e., 12-h post to $5 \mathrm{~min}, 24 \mathrm{~h}$ to $12 \mathrm{~h}$, etc.) that yielded a correlation value (Pearson's $R^{2}$ ) at each time-point after baseline. Irrespective of conscious intent, there was greater than chance carryover of pattern-specific chunks that did not increase over training and testing times. (C) Concatenation of chunks. As formed chunks concatenated into longer chunks, the ratio of maximum motor chunk length to total number of items within motor chunks should increase and approach one (fully concatenated). Here, if an item was fast (F) in at least six of eight repetitions (at least $75 \%$ ), it was counted as within a motor chunk, and we determined the ratio between the longest consecutive train of within chunk items and the total number of them. Irrespective of conscious intent, concatenation of pattern-specific chunks remained at chance levels.

\section{Acknowledgments}

This work was supported by the Intramural Research Program of the National Institute of Neurological Disorders and Stroke at the National Institutes of Health and utilized the high-performance computational capabilities of the Biowulf Linux cluster at the National Institutes of Health (http://biowulf.nih.gov).

\section{References}

Bo J, Borza V, Seidler RD. 2009. Age-related declines in visuospatial working memory correlate with deficits in explicit motor sequence learning. $J$ Neurophysiol 102: 2744-2754.

Boyd L, Winstein C. 2006. Explicit information interferes with implicit motor learning of both continuous and discrete movement tasks after stroke. J Neurol Phys Ther 30: 46-57.

Goedert KM, Willingham DB. 2002. Patterns of interference in sequence learning and prism adaptation inconsistent with the consolidation hypothesis. Learn Mem 9: 279-292.

Graybiel AM. 1998. The basal ganglia and chunking of action repertoires. Neurobiol Learn Mem 70: $119-136$

Jimenez L. 2008. Taking patterns for chunks: Is there any evidence of chunk learning in continuous serial reaction-time tasks? Psychol Res 72: 387-396

Keele SW, Ivry R, Mayr U, Hazeltine E, Heuer H. 2003. The cognitive and neural architecture of sequence representation. Psychol Rev 110: 316-339.

Kennerley SW, Sakai K, Rushworth MF. 2004. Organization of action sequences and the role of the pre-SMA. J Neurophysiol 91: 978-993.

Koch I, Hoffmann J. 2000. Patterns, chunks, and hierarchies in serial reaction-time tasks. Psychol Res 63: 22-35.

Lashley KS. 1951. The problem of serial order in behavior. In Cerebral mechanisms in behavior (ed. Jeffress LA), pp. 112-131. Wiley, New York.

Miller GA. 1956. The magical number seven plus or minus two: some limits on our capacity for processing information. Psychol Rev 63: 81-97.

Murray EA, Wise SP, Rhodes SEV. 2011. What can different brains do with reward? In Neurobiology of sensation and reward (ed. Gottfried JA). CRC Press, Boca Raton, FL.

Perruchet P, Vinter A. 2002. The self-organizing consciousness. Behav Brain Sci 25: 297-330.

Perruchet P, Vinter A, Pacteau C, Gallego J. 2002. The formation of structurally relevant units in artificial grammar learning. QJ Exp Psychol A 55: $485-503$.

Robertson EM, Pascual-Leone A, Press DZ. 2004. Awareness modifies the skill-learning benefits of sleep. Curr Biol 14: 208-212.

Rosenbaum DA, Kenny SB, Derr MA. 1983. Hierarchical control of rapid movement sequences. J Exp Psychol Hum Percept Perform 9: 86-102.

Sakai K, Kitaguchi K, Hikosaka O. 2003. Chunking during human visuomotor sequence learning. Exp Brain Res 152: 229-242.

Skaggs W, McNaughton BL. 1996. Replay of neuronal firing sequences in rat hippocampus during sleep following spatial experience. Science 271: $1870-1873$

Song S. 2009. Consciousness and the consolidation of motor learning. Behav Brain Res 196: $180-186$.

Song S, Cohen LG. 2014a. Practice and sleep form different aspects of skill. Nat Commun 5: 3407 .

Song S, Cohen LG. 2014b. Conscious recall of different aspects of skill memory. Front Behav Neurosci 9: 233.

Song S, Howard JH Jr, Howard DV. 2007a. Implicit probabilistic sequence learning is independent of explicit awareness. Learn Mem 14: $167-176$

Song S, Howard JH Jr, Howard DV. 2007b. Sleep does not benefit probabilistic motor sequence learning. J Neurosci 27: 12475-12483.

Song S, Marks B, Howard JH Jr, Howard DV. 2009. Evidence for parallel explicit and implicit sequence learning systems in older adults. Behav Brain Res 196: 328-332.

Song S, Sharma N, Buch ER, Cohen LG. 2012. White matter microstructural correlates of superior long-term skill gained implicitly under randomized practice. Cereb Cortex 7: 1671-1677.

Spencer RM, Sunm M, Ivry RB. 2006. Sleep-dependent consolidation of contextual learning. Curr Biol 16: 1001-1005.

Wymbs NF, Bassett DS, Mucha PJ, Porter MA, Grafton ST. 2012. Differential recruitment of the sensorimotor putamen and frontoparietal cortex during motor chunking in humans. Neuron 74: 936-946.

Received May 21, 2014; accepted in revised form June 27, 2014 


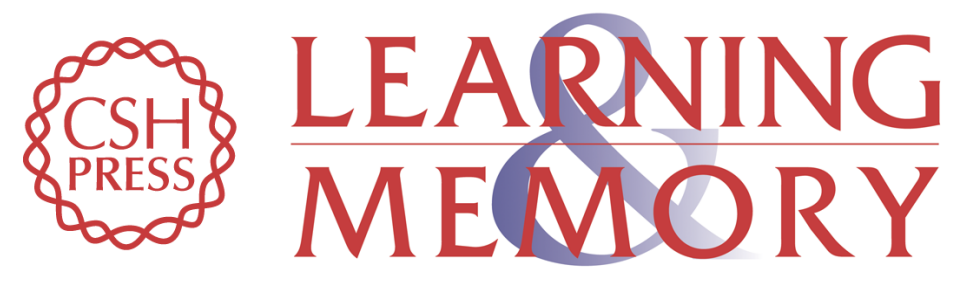

\section{Impact of conscious intent on chunking during motor learning}

Sunbin Song and Leonardo Cohen

Learn. Mem. 2014, 21:

Access the most recent version at doi:10.1101//m.035824.114

Supplemental http://learnmem.cshlp.org/content/suppl/2014/08/04/21.9.449.DC1
Material

References This article cites 24 articles, 4 of which can be accessed free at: http://learnmem.cshlp.org/content/21/9/449.full.html\#ref-list-1

Creative This article is distributed exclusively by Cold Spring Harbor Laboratory Press for the Commons License first 12 months after the full-issue publication date (see http://learnmem.cshlp.org/site/misc/terms.xhtml). After 12 months, it is available under a Creative Commons License (Attribution-NonCommercial 4.0 International), as described at http://creativecommons.org/licenses/by-nc/4.0/.

Email Alerting Receive free email alerts when new articles cite this article - sign up in the box at the Service top right corner of the article or click here. 